EPJ manuscript No.

(will be inserted by the editor)

\title{
Geometric study for the Legendre duality of generalized entropies and its application to the porous medium equation
}

\author{
Atsumi Ohara \\ Department of Systems Science, Osaka University, 1-3 Machikane-yama, Toyonaka, Osaka, 560-8531 JAPAN \\ e-mail: ohara@sys.es.osaka-u.ac.jp \\ Received: / Revised version:
}

\begin{abstract}
We geometrically study the Legendre duality relation that plays an important role in statistical physics with the standard or generalized entropies. For this purpose, we introduce dualistic structure defined by information geometry, and discuss concepts arising in generalized thermostatistics, such as relative entropies, escort distributions and modified expectations. Further, a possible generalization of these concepts in a certain direction is also considered. Finally, as an application of such a geometric viewpoint, we briefly demonstrate several new results on a behavior of the solution to the nonlinear diffusion equation called the porous medium equation.
\end{abstract}

PACS. 89.70.Cf Entropy and other measures of information - 02.40.Hw Classical differential geometry 05.90. $+\mathrm{m}$ Other topics in statistical physics, thermodynamics, and nonlinear dynamical systems

\section{Introduction}

In recent decades study of physical or artificial systems not obeying the usual Boltzmann-Gibbs statistical mechanics has received an increasing attention. For examples of such systems see $12 / 234$ and the references therein. Their common nature would be that the Boltzmann distribution does not correspond to their equilibriums. One of main research directions to overcome the difficulty of analysis for such systems is generalizing the notion of entropies within the framework of statistical physics.

In this generalization, the Legendre duality relation is still of fundamental importance and is required to prescribe a link between intensive and extensive parameters. In statistics this nice structure has been well exploited via information geometry [5]6] for the standard exponential family, and the results are successfully applied mainly to statistical estimation, information theory, learning theory and so on.

The purpose of this paper is to study the Legendre duality relation of generalized entropies from information geometric viewpoints and provide new insights and tools with this field by showing their usefulness through several applications.

In the aspect of geometric structure with Legendre duality, there may be at least two major directions to generalize the notion of entropies from the standard one. In section 2 we characterize the difference of these two methods in terms of a pair of representing functions for distributions. The one method always fixes one of representing

Send offprint requests to: functions to the identity map while the other varies both. The former leads to the geometry induced by the Bregman divergence. The latter includes the $\alpha$-geometry as a special case.

From section 3 to 7 , we discuss the Tsallis statistics, its generalization and applications. Section 3 presents a short review of the relation of the $\alpha$-geometry with the Tsallis statistics and emphasizes its importance. In section 4 we reconsider the construction of the $\alpha$-geometry by the affine surface theory [78|9] as preparation to a more generalized setup. The geometrical relation of two manifolds of the ordinary and the escort distributions are discussed. Section 5 proposes a generalization of the $\alpha$-geometric structure and the associated divergences using a certain class of convex functions. It is seen that the centro-affine immersion 9 is essential to conserve the dualistic structure. In section 6 and 7. we demonstrate applications of the introduced geometric notions to exploit the properties of generalized entropies. Section 6 shows the relation between modified averages (expectations) and convexities, which plays an important role in minimizing relative entropies under the average constraints. In section 7 , we prove that the trajectory of the gradient flow for the $\alpha$-divergence is a geodesic curve and it possesses several constants of motion.

Finally in sections 8 and 9 , we introduce the so-called Bregman divergence [10, the associated generalized entropy and geometry behind these quantities studied in [11]2 13]14. The feature is that the linear averages of the extensive physical quantities naturally appear in this setup. As an application, we show several new results on the behavior of the solutions to the porous medium equation (PME). The behavior is characterized in terms of 
A. Ohara: Geometric study for the Legendre duality of generalized entropies and its application.

geometric concepts induced on a generalized exponential family called the $q$-Gaussian densities. This family can be proved an invariant manifold for the PME. Further, we show that the trajectory of the solution on the manifold coincides with a geodesic curve with respect to the one of the dual affine connections. In addition the convergence rate to the manifold is evaluated. For this part, a full description with complete proofs can be found in [16].

\section{Statistical model and dualistic structure}

Let $p_{\zeta}(x)=p(x ; \zeta)$ be a probability distribution for random variable $x$ (or, density function for the continuous random variable) parametrized by a finite-dimensional parameter vector $\zeta=\left(\zeta^{1}, \cdots, \zeta^{n}\right) \in \mathcal{Z}$, where $\mathcal{Z}$ is a certain domain in $\mathbf{R}^{n}$. We call the set of $p_{\zeta}$ the statistical model and denote it by $\mathcal{M}$. The concrete examples in this paper are the probability simplex (3) and the $q$-Gaussian densities (44). We usually assume that $\mathcal{M}$ satisfies several regularity conditions such as smoothness of the map $\zeta \mapsto p_{\zeta}$, commutativity of integrations and differentiations and so on. See 5,6 for details.

It is known that the standard Boltzmann-GibbsShannon (BGS) entropy is maximized on the the Boltzmann distribution (exponential family) with the expectation constraint of the Hamiltonian. Similarly, each generalized entropy is maximized on the corresponding statistical model with the constraint. See, for example, Remark 1 in section 8. This is the major reason that motivates us to study structure of specific statistical models focusing on the Legendre duality of generalized entropies.

Information geometry is a convenient framework for this purpose. In order to introduce the geometric structure on the statistical model, we define the following quantities:

$$
\begin{aligned}
g_{i j}(\zeta) & :=\int \frac{\partial L\left(p_{\zeta}\right)}{\partial \zeta^{i}} \frac{\partial L^{*}\left(p_{\zeta}\right)}{\partial \zeta^{j}} d x, \\
\Gamma_{i j, k}(\zeta) & :=\int \frac{\partial^{2} L\left(p_{\zeta}\right)}{\partial \zeta^{i} \partial \zeta^{j}} \frac{\partial L^{*}\left(p_{\zeta}\right)}{\partial \zeta^{k}} d x, \\
\Gamma_{i j, k}^{*}(\zeta) & :=\int \frac{\partial L\left(p_{\zeta}\right)}{\partial \zeta^{k}} \frac{\partial^{2} L^{*}\left(p_{\zeta}\right)}{\partial \zeta^{i} \partial \zeta^{j}} d x .
\end{aligned}
$$

Here, a pair of one-to-one and smooth functions $L(u)$ and $L^{*}(u)$ on $u \geq 0$ are called representing functions for the distribution $p_{\zeta}$, which determines the Legendre duality such as pairs of dual coordinate systems (physically, extensive and intensive parameters) or potential functions conjugate each other. We use $g:=\left(g_{i j}\right)$ as a Riemannian metric on $\mathcal{M}$ and

$$
\Gamma_{i j}^{k}:=\sum_{l=1}^{n} g^{k l} \Gamma_{i j, l}, \quad \Gamma_{i j}^{* k}:=\sum_{l=1}^{n} g^{k l} \Gamma_{i j, l}^{*}
$$

as the components for two affine connections $\nabla$ and $\nabla^{*}$ on $\mathcal{M}$, where $g^{i j}$ is the component of the inverse matrix of $g$. Then the above definitions imply that the following duality relation of the connections [5]6] holds:

$$
\partial_{i} g_{j k}=\Gamma_{i j, k}+\Gamma_{i k, j}^{*},
$$

which is equivalent with (54) in Appendix A. This relation is important for the geometric study of the Legendre duality.

The typical cases are classified as follows:

i) The standard information geometry corresponding to the BGS entropy and Kullback-Leibler relative entropy is derived by

$$
L(u)=u, \quad L^{*}(u)=\ln u .
$$

ii) The $\alpha$-geometry corresponding to the Havrda-CharvatTsallis entropy (4) and Tsallis relative entropy (6) utilizes

$$
L(u)=L^{(\alpha)}(u):=\frac{2}{1-\alpha} u^{(1-\alpha) / 2}, \quad L^{*}(u)=L^{(-\alpha)}(u) .
$$

This class, its generalization and applications are discussed from section 3 to 7.

iii) Information geometry called the U-geometry 11 is corresponding to the Bregman-type divergences [11|12 13 14 15] and the associated generalized entropies. It is reproduced from

$$
L(u)=u, \quad L^{*}(u)=\ln _{\phi}(u),
$$

where $\ln _{\phi}$ is a generalized logarithmic function defined by, e.g., (32) 13 14 15. This class and its applications are discussed in section 8 and 9 .

Because $L(u)=u$ in the cases i) and iii), the linear average naturally appears and plays an important role. In these cases, the corresponding connections $\nabla$ and $\nabla^{*}$ are called (generalized) mixture and exponential connections, respectively. On the other hand, in the cases i) and ii) the obtained Riemannian metric $g$ coincides with the Fisher information, i.e.,

$$
g_{i j}(\zeta)=\int p_{\zeta} \frac{\partial \ln p_{\zeta}}{\partial \zeta^{i}} \frac{\partial \ln p_{\zeta}}{\partial \zeta^{j}} d x .
$$

This is a very important point in applying the geometry to statistical inference. We see from (2) that the relation

$$
\frac{d L}{d u} \frac{d L^{*}}{d u}=\frac{1}{u}
$$

should be satisfied for $g$ to be the Fisher information matrix. Hence, the Riemannian metric in the case iii) is not generally the Fisher metric. However, it is physically interpreted as a susceptance matrix via the linear average (See section 8).

\section{Review of Tsallis entropy via alpha-geometry}

Let $\mathcal{S}^{n}$ denote the $n$-dimensional probability simplex, i.e.,

$$
\mathcal{S}^{n}:=\left\{\boldsymbol{p}=\left(p_{i}\right) \mid p_{i}>0, \sum_{i=1}^{n+1} p_{i}=1\right\}
$$


and $p_{i}, i=1, \cdots, n+1$ denote probabilities of $n+1$ states. The set $\mathcal{S}^{n}$ is an example of the statistical model with parameters $p_{i}, i=1, \cdots, n$. The function $S_{q}$ defined on $\overline{\mathcal{S}}^{n}$, the closure of $\mathcal{S}^{n}$, for a real parameter $q(\neq 0$ nor 1$)$ by

$$
\begin{aligned}
S_{q}(\boldsymbol{p}) & :=-k \sum_{i=1}^{n+1}\left(p_{i}\right)^{q} \ln _{q} p_{i} \\
& =\frac{k}{1-q}\left(\sum_{i=1}^{n+1}\left(p_{i}\right)^{q}-1\right)
\end{aligned}
$$

is called the Havrda-Charvat-Tsallis (HCT) entropy 1718 , where $\ln _{q}$ is the $q$-logarithmic function defined by $\ln _{q} x=$ $\left(x^{1-q}-1\right) /(1-q)$. Note that the HCT entropy converges to the BGS entropy when $q$ goes to one. Hereafter, the positive constant $k$ is set to one for the sake of simplicity. The HCT entropy is concave if $q>0$ and convex if $q<0$. It does not satisfy the additivity, i.e., it holds that

$$
S_{q}(\boldsymbol{p} \otimes \boldsymbol{r})=S_{q}(\boldsymbol{p})+S_{q}(\boldsymbol{r})+(1-q) S_{q}(\boldsymbol{p}) S_{q}(\boldsymbol{r}) .
$$

for $\boldsymbol{p}=\left(p_{i}\right) \in \overline{\mathcal{S}}^{n}, \boldsymbol{r}=\left(r_{j}\right) \in \overline{\mathcal{S}}^{m}$ and $\boldsymbol{p} \otimes \boldsymbol{r}:=\left(p_{i} r_{j}\right) \in$ $\overline{\mathcal{S}}^{n m+n+m}$. The relation (15) is called nonextensivity. See, for details, a recent review paper 2 .

For several reasons the following quantity $K_{q}$ is introduced in [19 20 21 22] as a relative entropy between two probability distributions $\boldsymbol{p}$ and $\boldsymbol{r}$ in $\mathcal{S}^{n}$, which is of the form:

$$
\begin{aligned}
K_{q}(\boldsymbol{p}, \boldsymbol{r}) & :=-\sum_{i=1}^{n+1} p_{i} \ln _{q}\left(\frac{r_{i}}{p_{i}}\right) \\
& =\frac{1}{1-q}\left(1-\sum_{i=1}^{n+1}\left(p_{i}\right)^{q}\left(r_{i}\right)^{1-q}\right) .
\end{aligned}
$$

When $q$ is positive, $K_{q}(\boldsymbol{p}, \boldsymbol{r}) \geq 0$ and the equality holds if and only if $\boldsymbol{p}=\boldsymbol{r}$. Note that $K_{q}$ converges to the KullbackLeibler divergence as $q$ goes to one. For the uniform distribution $\boldsymbol{u}=\left(u_{i}\right)$ with $u_{i}=1 /(n+1)$ for all $i=1, \cdots n+1$, it holds

$$
K_{q}(\boldsymbol{p}, \boldsymbol{u})=\left(\frac{1}{n+1}\right)^{1-q}\left\{S_{q}(\boldsymbol{u})-S_{q}(\boldsymbol{p})\right\} .
$$

Hence, if $q>0$, the maximizing the HCT entropy $S_{q}(\boldsymbol{p})$ is equivalent to minimizing $K_{q}(\boldsymbol{p}, \boldsymbol{u})$.

On the other hand, the quantity called the $\alpha$-divergence [56] $D^{(\alpha)}$ has been used in mathematical statistics, which is defined with a real parameter $\alpha(\neq \pm 1)$ by

$$
D^{(\alpha)}(\boldsymbol{p}, \boldsymbol{r}):=\frac{4}{1-\alpha^{2}}\left\{1-\sum_{i=1}^{n+1}\left(p_{i}\right)^{(1-\alpha) / 2}\left(r_{i}\right)^{(1+\alpha) / 2}\right\}
$$

for two probabilities $\boldsymbol{p}$ and $\boldsymbol{r}$ in $\mathcal{S}^{n}$. By equating

$$
q:=(1-\alpha) / 2,
$$

we see that the Tsallis relative entropy $K_{q}$ coincides with the $\alpha$-divergence on $\mathcal{S}^{n}$ up to constant, i.e,

$$
D^{(\alpha)}(\boldsymbol{p}, \boldsymbol{r})=\frac{1}{q} K_{q}(\boldsymbol{p}, \boldsymbol{r}) .
$$

Note that $D^{(\alpha)}$ is nonnegative regardless to $\alpha$ and positive if and only if $\boldsymbol{p} \neq \boldsymbol{r}$. It also converges to the KullbackLeibler divergence when $\alpha \rightarrow-1$ and it is convex with respect to $\boldsymbol{p}$ and $\boldsymbol{r}$ if $-1<\alpha<1$.

It is known that the $\alpha$-divergence induces a differential geometric structure on $\mathcal{S}^{n}$ with a Riemannian metric and an affine connection denoted by $g$ and $\nabla^{(\alpha)}$, respectively. We call it the $\alpha$-geometry 56]. While the way to induce from the $\alpha$-divergence is omitted here, the resultant componentwise expressions of $g$ and $\nabla^{(\alpha)}$ are as follows: Let $\partial_{i}$ be a natural basis tangent vector field on $\mathcal{S}^{n}$ defined by

$$
\partial_{i}:=\frac{\partial}{\partial p_{i}}-\frac{\partial}{\partial p_{n+1}}, \quad i=1, \cdots, n
$$

Then, the induced Riemannian metric is nothing but the Fisher information matrix, i.e.,

$$
\begin{aligned}
g_{i j}(\boldsymbol{p}) & :=g\left(\partial_{i}, \partial_{j}\right)=\frac{1}{p_{i}} \delta_{i j}+\frac{1}{p_{n+1}} \\
& =\sum_{k=1}^{n+1} p_{k} \frac{\partial \log p_{k}}{\partial p_{i}} \frac{\partial \log p_{k}}{\partial p_{j}}, \quad i, j=1, \cdots, n .
\end{aligned}
$$

The induced affine connection $\nabla^{(\alpha)}$ is called the $\alpha$-connection, which is represented in its coefficients by

$$
\Gamma_{i j}^{(\alpha) k}(\boldsymbol{p})=\frac{1+\alpha}{2}\left(-\frac{1}{p_{k}} \delta_{i j}^{k}+p_{k} g_{i j}\right), \quad i, j, k=1, \cdots, n,
$$

where $\delta_{i j}^{k}$ is equal to one if $i=j=k$ and zero otherwise. Then we have its covariant derivatives by

$$
\nabla_{\partial_{i}}^{(\alpha)} \partial_{j}=\sum_{k=1}^{n} \Gamma_{i j}^{(\alpha) k} \partial_{k}
$$

There are two specific features for the $\alpha$-geometry on $\mathcal{S}^{n}$ induced in such a way. First, the triple $\left(\mathcal{S}^{n}, g, \nabla^{(\alpha)}\right)$ is a statistical manifold (See Appendix for its definition), i.e., we can confirm that the following holds:

$$
X g(Y, Z)=g\left(\nabla_{X}^{(\alpha)} Y, Z\right)+g\left(Y, \nabla_{X}^{(-\alpha)} Z\right)
$$

for arbitrary vector fields $X, Y$ and $Z$ on $\mathcal{S}^{n}$. Thus, $\nabla^{(\alpha)}$ and $\nabla^{(-\alpha)}$ are mutually dual. The relation (12) is closely related with the Legendre duality.

Another feature is that $\left(\mathcal{S}^{n}, g, \nabla^{(\alpha)}\right)$ is a manifold with constant curvature $\kappa=\left(1-\alpha^{2}\right) / 4=q(1-q)$, i.e., it holds that

$$
R^{(\alpha)}(X, Y) Z=\kappa\{g(Y, Z) X-g(X, Z) Y\},
$$

where $R^{(\alpha)}$ is the Riemann-Christoffel curvature with respect to $\nabla^{(\alpha)}$. Because of this property the $\alpha$-divergence meets the modified Pythagorean relation for $\boldsymbol{p}, \boldsymbol{q}$ and $\boldsymbol{r}$, which form a "right triangle" on $\mathcal{S}^{n}$ with respect to $g$ and $\nabla^{( \pm \alpha)}$, i.e.,

Proposition 1 Let $\gamma^{(\alpha)}$ and $\gamma^{(-\alpha)}$ be respectively the $\nabla^{(\alpha)}$ geodesic joining $\boldsymbol{p}$ and $\boldsymbol{q}$, and the $\nabla^{(-\alpha)}$-geodesic joining 
$\boldsymbol{q}$ and $\boldsymbol{r}$. If $\gamma^{(\alpha)}$ and $\gamma^{(-\alpha)}$ are orthogonal at $\boldsymbol{q}$ with respect to $g$, then it holds

$$
\begin{aligned}
D^{(\alpha)}(\boldsymbol{p}, \boldsymbol{r})= & D^{(\alpha)}(\boldsymbol{p}, \boldsymbol{q})+D^{(\alpha)}(\boldsymbol{q}, \boldsymbol{r}) \\
& -\kappa D^{(\alpha)}(\boldsymbol{p}, \boldsymbol{q}) D^{(\alpha)}(\boldsymbol{q}, \boldsymbol{r}) .
\end{aligned}
$$

This relation is quite important in studying the properties of the HCT entropy $S_{q}$ because its nonextensivity (5) is straightforwardly derived from (13). It means that nonflatness $(\kappa \neq 0)$ of the manifold $\mathcal{S}^{n}$ is geometrically interpreted as a direct cause of the nonextensivity 23. Further, (13) ensures the uniqueness of the equilibrium distribution minimizing the Tsallis relative entropy $K_{q}$ with constraints given in terms of the normalized $q$-expectation [23]. (See also the discussion in section 6])

\section{Escort distribution from a viewpoint of affine immersion}

In the previous section the $\alpha$-geometry is introduced from the $\alpha$-divergence. Another way to construct the $\alpha$-geometry on $\mathcal{S}^{n}$ is using the affine immersion 9 of $\mathcal{S}^{n}$ into $\mathbf{R}^{n+1}$ equipped with the standard flat connection $D$. The advantage of this method is that the escort probability naturally appears accompanying with the setup and its geometrical meaning is elucidated. Hereafter, we assume that $1>q=(1-\alpha) / 2>0$ to simplify the discussion. Several concepts of affine immersion are summarized in the appendix. For detail, see the references.

Let $\boldsymbol{\theta}=\left(\theta^{i}\right), i=1, \cdots, n+1$ be the standard coordinate system of the vector space $\mathbf{R}^{n+1}$ with respect to $\left\{o ; e_{1}, \cdots, e_{n+1}\right\}$, the origin as zero vector and the natural basis vectors. Denote by $\mathbf{R}_{+}^{n+1}$ the positive orthant of $\mathbf{R}^{n+1}$.

Consider the immersion $f$ of $\mathcal{S}^{n}$ into $\mathbf{R}_{+}^{n+1}$ by

$$
f: \boldsymbol{p}=\left(p_{i}\right) \mapsto \boldsymbol{\theta}=\left(\theta^{i}\right)=\left(L^{(\alpha)}\left(p_{i}\right)\right), \quad i=1, \cdots, n+1,
$$

where the representing function $L^{(\alpha)}$ is defined by

$$
L^{(\alpha)}(t):=\frac{2}{1-\alpha} t^{(1-\alpha) / 2}=\frac{1}{q} t^{q} .
$$

Note that $f\left(\mathcal{S}^{n}\right)$ is a level surface $\psi(\boldsymbol{\theta})=2 /(1+\alpha)$ in $\mathbf{R}_{+}^{n+1}$ of the function defined by

$\psi(\boldsymbol{\theta}):=\frac{2}{\alpha+1} \sum_{i=1}^{n+1}\left(\frac{1-\alpha}{2} \theta^{i}\right)^{2 /(1-\alpha)}=\frac{1}{1-q} \sum_{i=1}^{n+1}\left(q \theta^{i}\right)^{1 / q}$.

By the assumption for the range of $q=(1-\alpha) / 2$, the function $\psi$ is convex with the positive definite Hessian matrix on $\mathbf{R}_{+}^{n+1}$.

At this stage, we still have a freedom of choosing the transversal vector $\xi$. The freedom induces realizations of the different geometric structure of $\left(\mathcal{S}^{n}, h, \nabla\right)$. Here we take $\xi$ as

$$
\xi:=\sum_{i=1}^{n+1} \xi^{i} \frac{\partial}{\partial \theta^{i}}, \quad \xi^{i}=-(1-q) q \theta^{i}
$$

This choice of $\xi$ is derived by

$$
\xi=-\frac{1}{d \psi(E)} E=-\frac{1}{\sum_{i=1}^{n+1}\left(\partial \psi / \partial \theta^{i}\right) E^{i}} E,
$$

where $E=\sum_{i=1}^{n+1} E^{i} \partial / \partial \theta^{i}$ is the vector field defined to satisfy

$$
\frac{\partial^{2} \psi}{\partial \theta^{i} \partial \theta^{j}} X^{i} E^{j}=\sum_{i=1}^{n+1} \frac{\partial \psi}{\partial \theta^{i}} X^{i}=d \psi(X)
$$

for an arbitrary vector field $X=\sum_{i=1}^{n+1} X^{i} \partial / \partial \theta^{i}$ on $\mathbf{R}_{+}^{n+1}$ Hence, if $X$ is tangent to $f(\mathcal{M})$, then the right-hand side of (19) vanishes. However, since the Hessian $\left(\partial^{2} \psi / \partial \theta^{i} \partial \theta^{j}\right)$ is positive definite, $E$ and $\xi$ are guaranteed transversal to $f(\mathcal{M})$. Further, we see from (17) that the immersion $(f, \xi)$ is centro-affine with a scaling constant $q(1-q)$.

As summarized in the appendix, the centro-affine immersion realizes a statistical manifold $\left(\mathcal{S}^{n}, h, \nabla\right)$ with constant curvature. Actually, keeping in mind the relations

$$
\left(p_{i}\right)^{q-1} \frac{\partial}{\partial \theta^{i}}=\frac{\partial}{\partial p_{i}}, \quad i=1, \cdots, n+1
$$

and

$$
D_{\frac{\partial}{\partial \theta^{i}}} \frac{\partial}{\partial \theta^{j}}=0, \quad i, j=1, \cdots, n+1,
$$

we have

$$
D_{\frac{\partial}{\partial p_{i}}} \frac{\partial}{\partial p_{j}}=(q-1) \frac{\delta_{i j}}{p_{i}} \frac{\partial}{\partial p_{j}} \quad i, j=1, \cdots, n+1 .
$$

Using these relations, we can calculate the Gauss and Weingarten formulas (Cf. Appendix) for $\partial_{i}$ defined in (9) and $\xi$ as follows:

$$
\begin{aligned}
D_{\partial_{i}} \partial_{j} & =(q-1)\left\{\frac{\delta_{i j}}{p_{i}} \frac{\partial}{\partial p_{i}}+\frac{1}{p_{n+1}} \frac{\partial}{\partial p_{n+1}}\right\} \\
& =\sum_{k=1}^{n} \Gamma_{i j}^{k} \partial_{k}+h_{i j} \xi \\
D_{\partial_{i}} \xi & =q(q-1)\left\{\frac{\partial}{\partial p_{i}}-\frac{\partial}{\partial p_{n+1}}\right\} \\
& =-\sum_{j=1}^{n} s_{i}^{j} \partial_{j} .
\end{aligned}
$$

Here, we have used the expression:

$$
\xi=-(1-q) \sum_{i=1}^{n+1} p_{i} \frac{\partial}{\partial p_{i}}
$$

which is equivalent to (17). Then, we see that the calculated $h_{i j}$ and $\Gamma_{i j}^{k}$ respectively coincide with (10) and (11), i.e., it holds that $h_{i j}=g_{i j}$ and $\Gamma_{i j}^{k}=\Gamma_{i j}^{(\alpha) k}$. Thus, the realized manifold coincides with $\left(\mathcal{S}^{n}, g, \nabla^{(\alpha)}\right)$. Further, the affine shape operator $S=\left(s_{i}^{j}\right)$ and transversal connection form $\tau$ are, respectively,

$$
s_{i}^{j}=(1-q) q \delta_{i}^{j}, \quad \tau=0 .
$$


By the property F3) in the appendix, these two relations show that the realized manifold $\left(\mathcal{S}^{n}, g, \nabla^{(\alpha)}\right)$ is a statistical manifold with constant curvature $\kappa=q(1-q)$.

This viewpoint clarifies the relation with the escort probability. Using the coordinates $\left(\theta^{i}\right)$, the escort probability $P_{i}$ is expressed [1] by

$$
P_{i}(\boldsymbol{p}):=\frac{\left(p_{i}\right)^{q}}{\sum_{i=1}^{n+1}\left(p_{i}\right)^{q}}=\frac{\theta^{i}(\boldsymbol{p})}{\sum_{i=1}^{n+1} \theta^{i}(\boldsymbol{p})} \quad i=1, \cdots, n+1
$$

Hence, the set of escort probability distributions $\boldsymbol{P}=\left(P_{i}\right)$ with positive $P_{i}$, is nothing but the probability simplex in the ambient space $\mathbf{R}_{+}^{n+1}$. We denote this set by $\mathcal{E}^{n}$. Recall, on the other hand, that the immersion $f\left(\mathcal{S}^{n}\right)$ is represented as a level surface of $\psi$ in $\mathbf{R}_{+}^{n+1}$ (See Figure 11). Thus, for each $\left(p_{i}\right)$, we can define a projection $\pi$ from

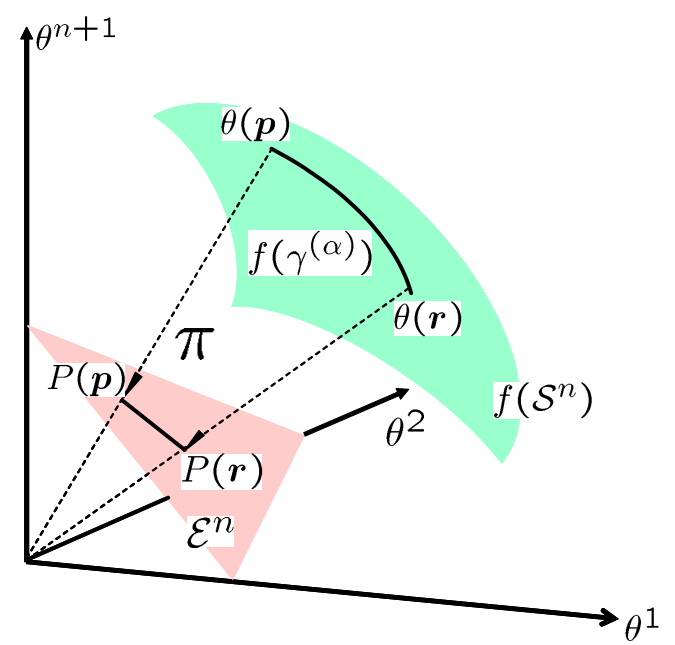

Fig. 1. Projective transformation $\pi$ from $f\left(\mathcal{S}^{n}\right)$ to $\mathcal{E}^{n}$ and the escort distribution $P$.

$f\left(\mathcal{S}^{n}\right)$ to $\mathcal{E}^{n}$ by

$$
\pi: f\left(\mathcal{S}^{n}\right) \ni \boldsymbol{\theta}=\left(\theta^{i}\right) \mapsto \boldsymbol{P}=\left(P_{i}\right)=\left(\lambda \theta^{i}\right) \in \mathcal{E}^{n},
$$

where

$$
\lambda:=\frac{1}{\sum_{i=1}^{n+1} \theta^{i}} .
$$

Now we consider geometric structure of $\mathcal{E}^{n}$ in order to derive interesting properties of the escort probabilities. Since $\mathcal{E}^{n}$ is contained in a hyperplane of $\mathbf{R}^{n+1}$, it would be natural to use the flat connection 1 induced from $D$. We use, for the brevity, the same symbol $D$ for the induced connection on $\mathcal{E}^{n}$. Then note that any straight line segment on $\mathcal{E}^{n}$ is a geodesic of $\left(\mathcal{E}^{n}, D\right)$.

Let $\mathcal{T}$ be the 2-dimensional sector defined by

$$
\mathcal{T}:=\left\{\boldsymbol{\theta} \mid \boldsymbol{\theta}=\beta_{1} \boldsymbol{\theta}(\boldsymbol{p})+\beta_{2} \boldsymbol{\theta}(\boldsymbol{r}), \beta_{1} \geq 0, \beta_{2} \geq 0\right\} .
$$

\footnotetext{
${ }^{1}$ It corresponds to the mixture connection 6 for the escort distributions $\left(P_{i}\right)$ in the terminology of information geometry.
}

Then it is known [9, p. 44] that the $\nabla^{(\alpha)}$-geodesic curve $\gamma^{(\alpha)}$ connecting $\boldsymbol{p}$ and $\boldsymbol{r}$ is represented on $f\left(\mathcal{S}^{n}\right)$ by

$$
f\left(\gamma^{(\alpha)}\right)=f\left(\mathcal{S}^{n}\right) \cap \mathcal{T} .
$$

On the other hand, $\mathcal{T}$ also includes points $\boldsymbol{P}(\boldsymbol{p})$ and $\boldsymbol{P}(\boldsymbol{r})$ by the definition of the escort probabilities. The intersection $\mathcal{E}^{n} \cap \mathcal{T}$ is a straight line segment, i.e, a geodesic of $(\mathcal{E}, D)$, connecting $\boldsymbol{P}(\boldsymbol{p})$ and $\boldsymbol{P}(\boldsymbol{r})$ (Figure 1).

Thus, $\pi \circ f$ maps every $\nabla^{(\alpha)}$-geodesic curve on $\left(\mathcal{S}^{n}, \nabla^{(\alpha)}\right)$ to a geodesic (line segment) on $\left(\mathcal{E}^{n}, D\right)$. In this sense, $\pi \circ f$ is a projective transformation from $\left(\mathcal{S}^{n}, \nabla^{(\alpha)}\right)$ to a flat manifold $\left(\mathcal{E}^{n}, D\right)$. The above observation is summarized as follows:

Proposition 2 The escort distribution $\left(P_{i}\right)$ is geometrically interpreted as a normalized affine coordinate system of the manifold $\mathcal{E}^{n}$ with the flat connection D, which is projectively transformed by $\pi \circ f$ from the probability simplex $\mathcal{S}^{n}$ with the connection $\nabla^{(\alpha)}$.

There are, at least, two possibilities A) and B) to introduce geometric structure to the flat manifold $\left(\mathcal{E}^{n}, D\right)$ equipped with the Legendre duality.

A) The one is to consider the Kullback-Leibler divergence, i.e., -1-divergence, for two escort distributions $\boldsymbol{P}=\left(P_{i}\right)$ and $\boldsymbol{P}^{\prime}=\left(P_{i}^{\prime}\right)$ on $\mathcal{E}^{n}$ :

$$
D^{(-1)}\left(\boldsymbol{P}, \boldsymbol{P}^{\prime}\right)=\sum_{i=1}^{n+1} P_{i} \ln \frac{P_{i}}{P_{i}^{\prime}}
$$

Then $\mathcal{E}^{n}$ is the well-developed dually flat statistical manifold [5]6] with the Fisher information matrix as a Riemannian metric. The obtained flat connection $D^{*}$ on $\mathcal{E}^{n}$ dual to $D$ is called the exponential connection. This structure is helpful when we apply the standard technique of statistical physics to the escort distributions (, e.g., [1]) and translate to the usual distributions.

B) The other possibility is to induce from the geometry of the ambient space $\mathbf{R}_{+}^{n+1}$ by regarding $\mathcal{E}^{n}$ as its submanifold. We do not describe the detail (See the note below), but we only show the corresponding divergence on $\mathcal{E}^{n}$.

Let $\varphi(\boldsymbol{\eta})$ be the Legendre transform of $\psi(\boldsymbol{\theta})$, i.e.,

$$
\begin{aligned}
\varphi(\eta) & =\sup _{\boldsymbol{\theta} \in \mathbf{R}_{+}^{n+1}}\left\{\sum_{i=1}^{n+1} \theta^{i} \eta_{i}-\psi(\boldsymbol{\theta})\right\} \\
& =\frac{2}{1-\alpha} \sum_{i=1}^{n+1}\left(\frac{1+\alpha}{2} \eta_{i}\right)^{2 /(1+\alpha)}, \eta_{i}:=\frac{\partial \psi}{\partial \theta^{i}}
\end{aligned}
$$

Construct the canonical divergence $\mathcal{D}$ [56] on $\mathbf{R}_{+}^{n+1} \times$ $\mathbf{R}_{+}^{n+1}$ using $\psi$ and the dual parameters $\eta_{i}$, then for two points $\boldsymbol{\theta}$ and $\boldsymbol{\theta}^{\prime}$ in $\mathbf{R}_{+}^{n+1}$ we have

$$
\begin{aligned}
\mathcal{D}\left(\boldsymbol{\theta}, \boldsymbol{\theta}^{\prime}\right) & :=\psi(\boldsymbol{\theta})+\varphi\left(\boldsymbol{\eta}^{\prime}\right)-\sum_{i=1}^{n+1} \theta^{i} \eta_{i}^{\prime} \\
& =\psi(\boldsymbol{\theta})-\psi\left(\boldsymbol{\theta}^{\prime}\right)-\sum_{i=1}^{n+1} \eta_{i}^{\prime}\left(\theta^{i}-\theta^{\prime i}\right) .
\end{aligned}
$$


The divergence $\mathcal{D}$ reproduces the $\alpha$-divergence (7) on $f\left(\mathcal{S}^{n}\right) \times$ $f\left(\mathcal{S}^{n}\right)$ due to the relations (14), (15) and

$$
\eta_{i}(\boldsymbol{p})=L^{(-\alpha)}\left(p_{i}\right)=\frac{1}{1-q}\left(p_{i}\right)^{1-q}, i=1, \cdots, n+1 .
$$

Simultaneously, it induces the following natural divergence for two escort distributions $\boldsymbol{P}=\left(P_{i}\right)$ and $\boldsymbol{P}^{\prime}=\left(P_{i}^{\prime}\right)$ on $\mathcal{E}^{n}$ :

$$
\mathcal{D}\left(\boldsymbol{P}, \boldsymbol{P}^{\prime}\right)=\psi(\boldsymbol{P})-\psi\left(\boldsymbol{P}^{\prime}\right)-\sum_{i=1}^{n+1}\left(P_{i}-P_{i}^{\prime}\right) P_{i}^{\prime *},
$$

where $P_{i}^{*}$ is defined by

$$
P_{i}^{*}:=\frac{1}{1-q}\left(q P_{i}\right)^{(1-q) / q} .
$$

Note: The canonical divergence (22) defines the flat statistical manifold structure $\left(\mathbf{R}_{+}^{n+1}, \tilde{g}, D\right)$ with the Riemannian metric $\tilde{g}$ :

$$
\begin{aligned}
\tilde{g}_{i j} & :=-\left.\frac{\partial}{\partial \theta^{i}} \frac{\partial}{\partial \theta^{\prime j}} \mathcal{D}\left(\boldsymbol{\theta}, \boldsymbol{\theta}^{\prime}\right)\right|_{\boldsymbol{\theta}=\boldsymbol{\theta}^{\prime}} \\
& =\frac{\partial \psi}{\partial \theta^{i} \partial \theta^{j}}=\left(q \theta^{i}\right)^{(1-2 q) / q} \delta_{i j} .
\end{aligned}
$$

and the flat connection $D$. Further, it induces a geometry on $\mathcal{S}^{n}$ via $f\left(\mathcal{S}^{n}\right)$ as the statistical submanifold of $\mathbf{R}_{+}^{n+1}$. This is the essentially same way to 23 or section 3 . We have seen in this section that the induced geometry coincides with the one defined by the affine immersion $(f, \xi)$. This is due to the special choice of the transversal vector $\xi$ satisfying (18) and (19). See for detail 24125/26].

The manifold $\left(\mathbf{R}_{+}^{n+1}, \tilde{g}, D\right)$ also induces geometry on the submanifold $\mathcal{E}^{n}$. It can be proved to be a flat statistical manifold and its corresponding divergence is given in (24). The induced geometry is known to be not only projectively but also conformally transformed by $\pi \circ f$ from $\left(\mathcal{S}^{n}, g, \nabla^{(\alpha)}\right)$, which directly follow from the concept of -1 -conformal equivalence 8 .

\section{Generalization via centro-affine immersion}

In this section we discuss a possible generalization of entropy, relative entropy (divergence), escort probability keeping statistical manifold structure of $\mathcal{S}^{n}$. The key idea is by applying the method of centro-affine immersion observed in the previous section.

Consider a smooth representing function $s=L(t)$ defined on $t \geq 0$ satisfying the following assumptions:

A1: strictly increasing,

A2: $L(0)=0$,

A3: $d^{2} L / d t^{2}<0$ for all $t \geq 0$.

These assumptions ensure that the strictly increasing inverse function $L^{-1}$ exists and meets conditions: $L^{-1}(0)=$ 0 and $d^{2} L^{-1} / d s^{2}>0$ for all $s \geq 0$. Hence, $L^{-1}$ is a convex function.
Let $f$ be an affine immersion defined by (14), then $\mathcal{S}^{n}$ is immersed in $\mathbf{R}_{+}^{n+1}$ as a level surface of $\psi^{(L)}$ :

$$
f\left(\mathcal{S}^{n}\right):=\left\{\boldsymbol{\theta} \mid \sum_{i=1}^{n+1} \psi^{(L)}(\boldsymbol{\theta})=c, \quad \exists c>0\right\} .
$$

Here, $\psi^{(L)}$ is a convex function defined by

$$
\psi^{(L)}(\boldsymbol{\theta}):=c \sum_{i=1}^{n+1} L^{-1}\left(\theta^{i}\right),
$$

which has a positive definite Hessian from the assumptions. The level $c$ is arbitrary. For the case of the $\alpha$ geometry we have used $L=L^{(\alpha)}$ and $c=2 /(1+\alpha)$.

We take a transversal vector $\xi$ so that the affine immersion $(f, \xi)$ is centro-affine with a scaling constant $\tilde{\kappa}$ :

$$
\xi=\sum_{i=1}^{n+1} \xi^{i} \frac{\partial}{\partial \theta^{i}}, \quad \xi^{i}=-\tilde{\kappa} \theta^{i}
$$

where $\tilde{\kappa}$ is an arbitrary constant. Since $f\left(\mathcal{S}^{n}\right)$ is a strongly convex surface, $\xi$ defined above is transversal to $f\left(\mathcal{S}^{n}\right)$. Hence, according to F3) in the appendix, we find that $(f, \xi)$ realizes a statistical manifold $\left(\mathcal{S}^{n}, h^{(L)}, \nabla^{(L)}\right)$ with constant curvature $\tilde{\kappa}$.

Instead of the canonical divergence, we invoke the geometric divergence [8]:

$$
D^{(L)}(\boldsymbol{p}, \boldsymbol{r}):=-\sum_{i=1}^{n+1} \nu_{i}(\boldsymbol{r})\left(\theta^{i}(\boldsymbol{p})-\theta^{i}(\boldsymbol{r})\right),
$$

where $\nu=\sum_{i=1}^{n+1} \nu_{i} d \theta^{i} \in\left(\mathbf{R}^{n+1}\right)^{*}$ is called the conormal vector [9] defined by

$$
\nu_{i}:=\frac{1}{\Lambda} \frac{\partial \psi^{(L)}}{\partial \theta^{i}}, i=1, \cdots, n+1,
$$

where

$$
\Lambda:=d \psi^{(L)}(\xi)=\sum_{i=1}^{n+1} \frac{\partial \psi^{(L)}}{\partial \theta^{i}} \xi^{i} .
$$

Here, $\left(\mathbf{R}^{n+1}\right)^{*}$ denotes the dual space of $\mathbf{R}^{n+1}$ and $\nu_{i}$ plays a similar role of the dual parameter $\eta_{i}$ in the case of the $\alpha$-geometry.

It is known 8 that the analogous statement to Proposition 1 holds for $D^{(L)}$, i.e., for a right triangle on $\mathcal{S}^{n}$ with respect to the realized Riemannian metric $h^{(L)}$ and dual connections $\nabla^{(L)}$ and $\nabla^{(L) *}$, it holds that

$$
\begin{aligned}
D^{(L)}(\boldsymbol{p}, \boldsymbol{r})= & D^{(L)}(\boldsymbol{p}, \boldsymbol{q})+D^{(L)}(\boldsymbol{q}, \boldsymbol{r}) \\
& -\tilde{\kappa} D^{(L)}(\boldsymbol{p}, \boldsymbol{q}) D^{(L)}(\boldsymbol{q}, \boldsymbol{r}) .
\end{aligned}
$$

Using the uniform distribution $\boldsymbol{u}$, we can define an associated entropy $S^{(L)}$ with the divergence $D^{(L)}$ by

$$
S^{(L)}(\boldsymbol{p}):=M-D^{(L)}(\boldsymbol{p}, \boldsymbol{u})
$$


where

$$
M:=\max _{\boldsymbol{p} \in \overline{\mathcal{S}}^{n}} D^{(L)}(\boldsymbol{p}, \boldsymbol{u}) .
$$

Then $S^{(L)}$ is confirmed to meet positivity, convexity, continuity and take the maximum at $\boldsymbol{p}=\boldsymbol{u}$. The modified Pythagorean relation (28) is essentially important to investigate the minimization of the generalized divergence $D^{(L)}$ or maximization of the generalized entropy $S^{(L)}$, as is in the case of $K_{q}$ or $S_{q}$ 23].

The generalized escort probability (see also [1]) is similarly defined by

$$
P_{i}^{(L)}:=\frac{L\left(p_{i}\right)}{\sum_{i=1}^{n+1} L\left(p_{i}\right)} .
$$

The projectivity and the property in Proposition 2 are inherited to this generalization.

However, unlike the case of the previous section, the divergence defined on $\mathbf{R}_{+}^{n+1}$ by (22) with $\psi^{(L)}$ instead of $\psi$ does not generally induce $D^{(L)}$ in (26) on $\mathcal{S}^{n}$. By comparing (22) and (26), we see that the property holds if and only if $\nu_{i}$ coincides with the dual parameter of $\theta^{i}$, i.e.,

$$
\nu_{i}=\frac{\partial \psi^{(L)}}{\partial \theta^{i}}, \quad i=1, \cdots, n+1 .
$$

From (27) it is equivalent to the condition that $\Lambda=1$, i.e.,

$$
d \psi^{(L)}(\xi)=\tilde{\kappa} \sum_{i=1}^{n+1} \frac{\partial \psi^{(L)}}{\partial \theta^{i}} \theta^{i}=1
$$

holds on $f\left(\mathcal{S}^{n}\right)$. Thus, the property is specific to the case $L$ is a power function like (15).

\section{Application (1): Alpha-convexity and autoparallelism of submanifolds constrained by expectations}

In this section, we apply the geometry $\left(\mathcal{S}^{n}, g, \nabla^{(\alpha)}\right)$ to discuss convexity of submanifolds constrained by the modified expectations (averages). Convexity of the submanifold is crucial in considering the maximization of $S_{q}$. Here we restrict to the case that $q>0, q \neq 1$. The results can be generalized to $\left(\mathcal{S}^{n}, h^{(L)}, \nabla^{(L)}\right)$ via the analogous arguments. that

The subset $\mathcal{A}$ in $\mathcal{S}^{n}$ is said $\nabla^{(\alpha)}$-autoparallel, if it holds

$$
f(\mathcal{A})=f\left(\mathcal{S}^{n}\right) \cap \mathcal{T}
$$

for a certain open convex set $\mathcal{T}$ contained in a linear subspace of $\mathbf{R}^{n+1}$ with respect to the $\theta$-coordinate system. The characterization of the $\nabla^{(\alpha)}$-geodesic curve of (20) is the special case where the dimension of the linear subspace is two. The subset $\mathcal{C}$ in $\mathcal{S}^{n}$ is said $\nabla^{(\alpha)}$-convex if the $\alpha$-geodesic curve connecting arbitrary two points on $\mathcal{C}$ is contained in $\mathcal{C}$. Note that $\mathcal{A}$ is $\nabla^{(\alpha)}$-convex if it is $\nabla^{(\alpha)}$-autoparallel.
The $\nabla^{(\alpha)}$-convexity or $\nabla^{(\alpha)}$-autoparallelism are important in studying the Tsallis relative entropy minimization from a viewpoint of the optimization. It is because the modified Pythagorean theorem guarantees the uniqueness of the minimizing distribution on the $\nabla^{(\alpha)}$-convex set. In this case, the minimizing distribution is characterized by the so-called $-\alpha$-projection $5[6] 23$.

Let $A_{i}$ be the quantity assigned to the $i$-th microstate and consider the linear, $q$ - and $q$-normalized expectations [27, respectively defined by

$$
\begin{gathered}
\langle A\rangle:=\sum_{i=1}^{n+1} p_{i} A_{i}, \quad\langle A\rangle_{q}:=\sum_{i=1}^{n+1}\left(p_{i}\right)^{q} A_{i}, \\
\langle\langle A\rangle\rangle_{q}:=\frac{\sum_{i=1}^{n+1}\left(p_{i}\right)^{q} A_{i}}{\sum_{i=1}^{n+1}\left(p_{i}\right)^{q}} .
\end{gathered}
$$

For a prescribed value $\bar{A}$, define the constrained manifolds in $\mathcal{S}^{n}$ by

$$
\begin{gathered}
\mathcal{H}^{+}:=\mathcal{S}^{n} \cap\{\boldsymbol{p} \mid\langle A\rangle \geq \bar{A}\}, \quad \mathcal{H}_{q}^{+}:=\mathcal{S}^{n} \cap\left\{\boldsymbol{p} \mid\langle A\rangle_{q} \geq \bar{A}\right\}, \\
\widetilde{\mathcal{H}}_{q}^{+}:=\mathcal{S}^{n} \cap\left\{\boldsymbol{p} \mid\langle\langle A\rangle\rangle_{q} \geq \bar{A}\right\} .
\end{gathered}
$$

Similarly, $\mathcal{H}^{-}, \mathcal{H}_{q}^{-}, \widetilde{\mathcal{H}}_{q}^{-}$, and the boundaries $\mathcal{H}, \mathcal{H}_{q}, \widetilde{\mathcal{H}}_{q}$ can be defined by replacing the inequality symbols by the reverse and the equality ones, respectively.

Since the constraints for $\mathcal{H}_{q}^{ \pm}, \widetilde{\mathcal{H}}_{q}^{ \pm}, \mathcal{H}_{q}$ or $\widetilde{\mathcal{H}}_{q}$ are nonlinear with respect to $\boldsymbol{p}$, the corresponding equilibrium distributions for the Tsallis relative entropy $K_{q}(\cdot, \boldsymbol{r})$ (or $\alpha$-divergence $\left.D^{(\alpha)}(\cdot, \boldsymbol{r})\right)$ are not necessarily unique nor the minimizers for them, while they are convex in the usual sense with respect to $\boldsymbol{p}$ when $q>0$ and $q \neq 1$.

Let the constrained subsets be nonempty. Then they have the following properties:

1) $\mathcal{H}^{-}$(resp. $\left.\mathcal{H}^{+}\right)$is $\nabla^{(\alpha)}$-convex if $0<q<1(q>1)$ and $A_{i}>0$ for all $i$,

2) $\mathcal{H}_{q}^{+}\left(\mathcal{H}_{q}^{-}\right)$is $\nabla^{(\alpha)}$-convex if $0<q<1(1<q)$,

3) Let $0<q<1(1<q)$ and $0<A_{i}\left(0>A_{i}\right)$ for all $i$. Then $\mathcal{H}_{q}$ is a $D^{(\alpha)}$-sphere, i.e.,

$$
\mathcal{H}_{q}=\mathcal{S}^{n} \cap\left\{\boldsymbol{p} \mid D^{(\alpha)}(\boldsymbol{p}, \boldsymbol{r})=d\right\}
$$

for some $\boldsymbol{r} \in \mathcal{S}^{n}$ and $d \in \mathbf{R}$,

4) Both $\widetilde{\mathcal{H}}_{q}^{ \pm}$are $\nabla^{(\alpha)}$-convex,

5) $\widetilde{\mathcal{H}}_{q}$ is a $\nabla^{(\alpha)}$-autoparallel submanifold in $\mathcal{S}^{n}$.

All of the above statements follow from the standard convexity argument with respect to the $\theta$-coordinate based on the definition of the $\alpha$-convexity. In particular, note that constraints $\langle A\rangle_{q}=\bar{A}$ and $\langle\langle A\rangle\rangle_{q}=\bar{A}$ are respectively characterized by linear constraints in $\theta$, i.e.,

$$
q \sum_{i=1}^{n+1} A_{i} \theta^{i}=\bar{A}, \quad \sum_{i=1}^{n+1}\left(A_{i}-\bar{A}\right) \theta^{i}=0 .
$$

For 3), recall that the $\alpha$-divergence can be alternatively expressed, using (23), by

$$
D^{(\alpha)}(\boldsymbol{p}, \boldsymbol{r})=\frac{1}{q(1-q)}-\sum_{i=1}^{n+1} \theta^{i}(\boldsymbol{p}) \eta_{i}(\boldsymbol{r}) .
$$


Then we can verify the statement by setting

$$
\eta_{i}(\boldsymbol{r}):=\epsilon^{q-1} A_{i}, \quad d:=\frac{1}{q}\left(\frac{1}{1-q}-\epsilon^{q-1} \bar{A}\right),
$$

where

$$
\epsilon:=\sum_{i=1}^{n+1}\left((1-q) A_{i}\right)^{1 /(1-q)} .
$$

For the detail of the Tsallis relative entropy minimization with the constraints of $\widetilde{\mathcal{H}}_{q}$, see [23].

\section{Application (2): Gradient flow of the alpha-divergence}

As another application we investigate the gradient flow of the $\alpha$-divergence. Let $X=\sum_{i=1}^{n} X^{i} \partial_{i}$ be the gradient vector on $\mathcal{S}^{n}$ minimizing $D^{(\alpha)}(\boldsymbol{p}, \boldsymbol{r})$ for a given distribution $\boldsymbol{r}$, where $\partial_{i}$ is defined by (9). Then the component $X^{i}$ is expressed by

$$
X^{i}=-\sum_{j=1}^{n} g^{i j} \partial_{j} D^{(\alpha)}(\boldsymbol{p}, \boldsymbol{r}), \quad i=1, \cdots, n,
$$

where $\left(g^{i j}\right)$ is the inverse matrix of the Riemannian metric $g=\left(g_{i j}\right)$ in (10).

Since $D^{(\alpha)}(\boldsymbol{p}, \boldsymbol{r})$ is strongly convex with respect to $\boldsymbol{p}$ in $\mathcal{S}^{n}$, the flow converges to $\boldsymbol{r}$. Further, the constant curvature property of $\left(\mathcal{S}^{n}, g, \nabla^{(\alpha)}\right)$ gives the restriction for the behavior of the gradient flow as follows. For dually flat case, the corresponding results are found in [28].

Proposition 3 (constants of motion) The trajectory of the gradient flow $\boldsymbol{p}(t)$ of $D^{(\alpha)}(\boldsymbol{p}, \boldsymbol{r})$ on $\left(\mathcal{S}^{n}, g, \nabla^{(\alpha)}\right)$ with an initial point $\boldsymbol{p}_{0}$ coincides with the $\nabla^{(-\alpha)}$-geodesic curve connecting $\boldsymbol{p}_{0}$ and $\boldsymbol{r}$. Further, let $\boldsymbol{\theta}_{l}=\left(\theta_{l}^{i}\right) \in \mathbf{R}^{n+1}, l=$ $1, \cdots, n-1$ be the set of linearly independent vectors satisfying

$$
\sum_{i=1}^{n+1} \theta_{l}^{i} \eta_{i}\left(\boldsymbol{p}_{0}\right)=0, \quad \sum_{i=1}^{n+1} \theta_{l}^{i} \eta_{i}(\boldsymbol{r})=0, \quad l=1, \cdots, n-1 .
$$

Then, the quantities $C_{l}$ defined by

$$
C_{l}:=\sum_{i=1}^{n+1} \theta_{l}^{i} \eta_{i}(\boldsymbol{p}(t)), \quad l=1, \cdots, n-1
$$

are the $n-1$ independent constants of motion for the gradient flow.

See appendix B for the proof.

\section{Generalized exponential family and U-geometry}

Now we discuss the Legendre duality in the case of iii) in section 2, following [1112131415]. For a fixed strictly increasing and positive function $\phi(s)$ on $(0, \infty)$, define the generalized logarithmic function as follows:

$$
\ln _{\phi}(t):=\int_{1}^{t} \frac{1}{\phi(s)} d s, \quad t>0 .
$$

The generalized exponential function denoted by $\exp _{\phi}$ is defined as the inverse function of $\ln _{\phi}$.

Define a convex function $F_{\phi}(s)$ for $s>0$ by

$$
F_{\phi}(s):=\int_{1}^{s} \ln _{\phi} t d t, \quad \lim _{s \rightarrow 0_{+}} F_{\phi}(s)<+\infty \text { :assumed. }
$$

For probability density functions $p(x)$ and $r(x)$, introduce a generalized entropy functional defined by

$$
\mathcal{I}_{\phi}[p]:=\int-F_{\phi}(p(x))+(1-p(x)) F_{\phi}(0) d x,
$$

and the Bregman divergence defined by

$$
\mathcal{D}_{\phi}[p \| r]:=\int U_{\phi}\left(\ln _{\phi} r\right)-U_{\phi}\left(\ln _{\phi} p\right)-p\left(\ln _{\phi} r-\ln _{\phi} p\right) d x,
$$

where the function $U_{\phi}$ is the Legendre conjugate of $F_{\phi}$ defined by

$$
U_{\phi}(t):=t \exp _{\phi} t-F_{\phi}\left(\exp _{\phi} t\right) .
$$

Let us consider the following finite dimensional statistical model called the generalized exponential family [29] ( $\phi$-exponential family [15] or $U$-statistical model [11]), which is defined by

$$
\mathcal{M}_{\phi}=\left\{p_{\theta}(x)=\exp _{\phi}\left(\theta^{T} h(x)-\psi_{\phi}(\theta)\right) \mid \theta \in \Omega \subset \mathbf{R}^{d}\right\}
$$

where $h(x)=\left(h_{i}(x)\right), i=1, \cdots, d$ is a certain vectorvalued function and $\psi_{\phi}(\theta)$ is a normalizing factor of $p_{\theta}(x)$.

Introduce the following potential function:

$$
\Psi_{\phi}(\theta):=\int U_{\phi}\left(\ln _{\phi} p_{\theta}\right)+\left(1-p_{\theta}\right) F_{\phi}(0) d x+\psi_{\phi}(\theta) .
$$

It follows from the relation $\exp _{\phi}=U_{\phi}^{\prime}$ that

$$
\eta_{i}(\theta):=\partial_{i} \Psi_{\phi}(\theta)=\int h_{i}(x) p_{\theta}(x) d x=\mathbf{E}_{p_{\theta}}\left[h_{i}(x)\right]
$$

where $\partial_{i}:=\partial / \partial \theta^{i}$ and we denote by $\mathbf{E}_{p}[\cdot]$ the expectation operator for the density $p$. Then, the Hesse matrix of $\Psi_{\phi}(\theta)$ is expressed by

$$
\partial_{i} \partial_{j} \Psi_{\phi}(\theta)=\int \tilde{h}_{i}(x) \exp _{\phi}^{\prime}\left(\theta^{T} h(x)-\psi_{\phi}(\theta)\right) \tilde{h}_{j}(x) d x
$$

where $\tilde{h}_{i}(x):=h_{i}(x)-\partial_{i} \psi_{\phi}(\theta)$. We see that it is positive semidefinite because $\exp _{\phi}^{\prime}$ is positive, and hence, $\Psi_{\phi}$ is a convex function of $\theta$. In the sequel, we assume that $\left(\partial_{i} \partial_{j} \Psi_{\phi}\right)=\left(\partial \eta_{j} / \partial \theta^{i}\right)$ is positive definite for $\forall \theta \in \Omega$. Hence, $\eta=\left(\eta_{i}\right)$ is locally bijective to $\left(\theta^{i}\right)$ and we call $\eta=\left(\eta_{i}\right)$ the expectation coordinate system for $\mathcal{M}_{\phi}$. By 
the relation (37) the Legendre conjugate of $\Psi_{\phi}(\theta)$ is the sign-reversed generalized entropy of $p_{\theta} \in \mathcal{M}_{\phi}$, i.e,

$$
\Psi_{\phi}^{*}(\eta)=\theta^{T} \eta-\Psi_{\phi}(\theta)=-\mathcal{I}_{\phi}\left[p_{\theta}\right] .
$$

Hence, $\Psi_{\phi}(\theta)$ can be physically interpreted as the generalized Massieu potential 3031 and our Riemannian metric $\left(\partial_{i} \partial_{j} \Psi_{\phi}\right)=\left(\partial \eta_{j} / \partial \theta^{i}\right)$ introduced below is regarded as a susceptance matrix.

The U-geometry [11 is introduced as follows: As a Riemannian metric $g=\left(g_{i j}\right)$ on $\mathcal{M}_{\phi}$, which is an inner product for tangent vectors, we use the Hesse matrix of $\Psi_{\phi}$. Note that we can alternatively express (38) as

$$
g_{i j}(\theta)=g\left(\partial_{i}, \partial_{j}\right):=\partial_{i} \partial_{j} \Psi_{\phi}=\int \partial_{i} p_{\theta} \partial_{j} \ln _{\phi} p_{\theta} d x
$$

Further we define a generalized version of the mixture connection $\nabla^{(\mathrm{m})}$ and exponential connection $\nabla^{(\mathrm{e})}$ by their components

$$
\begin{gathered}
\Gamma_{i j, k}^{(\mathrm{m})}(\theta)=g\left(\nabla_{\partial_{i}}^{(\mathrm{m})} \partial_{j}, \partial_{k}\right):=\int \partial_{i} \partial_{j} p_{\theta} \partial_{k} \ln _{\phi} p_{\theta} d x \\
\Gamma_{i j, k}^{(\mathrm{e})}(\theta)=g\left(\nabla_{\partial_{i}}^{(\mathrm{e})} \partial_{j}, \partial_{k}\right):=\int \partial_{k} p_{\theta} \partial_{i} \partial_{j} \ln _{\phi} p_{\theta} d x .
\end{gathered}
$$

Then the duality relation of the connections [5]6] holds, i.e., $\partial_{i} g_{j k}=\Gamma_{i j, k}^{(\mathrm{m})}+\Gamma_{i k, j}^{(\mathrm{e})}$. Further, $\mathcal{M}_{\phi}$ can be proved to be flat with respect to both $\nabla^{(\mathrm{m})}$ and $\nabla^{(\mathrm{e})}$. Thus, we have obtained dually flat 6 structure $\left(g, \nabla^{(\mathrm{m})}, \nabla^{(\mathrm{e})}\right)$ on $\mathcal{M}_{\phi}$ defined by the derivatives of $\Psi_{\phi}$.

Proposition 4 Let $\mathcal{C}$ be a one-dimensional submanifold on $\mathcal{M}_{\phi}$. If $\mathcal{C}$ is expressed as a straight line in the coordinates $\theta$, then $\mathcal{C}$ coincides with $a \nabla^{(\mathrm{e})}$-geodesic (e-geodesic, in short) curve. If $\mathcal{C}$ is expressed as a straight line in the coordinates $\eta$, then $\mathcal{C}$ coincides with a $\nabla^{(\mathrm{m})}$-geodesic $(\mathrm{m}-$ geodesic) curve.

Definition 1 Let $p(x)$ be a given density. If there exists the minimizing density function $\hat{p}_{\theta}(x)$ for the variational problem $\min _{p_{\theta} \in \mathcal{M}_{\phi}} \mathcal{D}_{\phi}\left[p \| p_{\theta}\right]$, or equivalently, the minimizing parameter $\hat{\theta}$ for the problem $\min _{\theta \in \Omega} \mathcal{D}_{\phi}\left[p \| p_{\theta}\right]$ exists, we call $\hat{p}_{\theta}(x)=p_{\hat{\theta}}(x)$ the m-projection of $p(x)$ to $\mathcal{M}_{\phi}$.

Proposition 5 Let $\hat{p}_{\theta} \in \mathcal{M}_{\phi}$ be the m-projection of $p$. Then the following properties hold:

i) The expectation of $h(x)$ is conserved by the m-projection, i.e., $\mathbf{E}_{p}[h(x)]=\mathbf{E}_{\hat{p}_{\theta}}[h(x)]$,

ii) The following triangular equality holds: $\mathcal{D}_{\phi}\left[p \| p_{\theta}\right]=$ $\mathcal{D}_{\phi}\left[p \| \hat{p}_{\theta}\right]+\mathcal{D}_{\phi}\left[\hat{p}_{\theta} \| p_{\theta}\right]$ for all $p_{\theta} \in \mathcal{M}_{\phi}$.

Remark 1 From the statement i) the m-projection $\hat{p}_{\theta}$ is characterized as the density in $\mathcal{M}_{\phi}$ with the equal expectation of $h(x)$ to that for $p$. Note that the following relation:

$$
\begin{aligned}
\mathcal{D}_{\phi}\left[p \| \hat{p}_{\theta}\right] & =\Psi_{\phi}(\hat{\theta})-\mathcal{I}_{\phi}[p]-\hat{\theta}^{T} \mathbf{E}_{p}[h(x)] \\
& =\Psi_{\phi}(\hat{\theta})-\hat{\theta}^{T} \hat{\eta}-\mathcal{I}_{\phi}[p]=\mathcal{I}_{\phi}\left[\hat{p}_{\theta}\right]-\mathcal{I}_{\phi}[p] \geq 0 .
\end{aligned}
$$

Thus, $\hat{p}_{\theta}$ achieves the maximum entropy among densities with the equal expectation of $h(x)$.

\section{Application (3) : Nonlinear diffusion equation}

Let $u(x, t)$ and $p(x, \tau)$ on $\mathbf{R}^{n} \times \mathbf{R}_{+}$be, respectively, the solutions of the following nonlinear diffusion equation, which is called the porous medium equation (PME):

$$
\frac{\partial u}{\partial t}=\Delta u^{m}, \quad m>1
$$

with nonnegative initial data $0 \leq u(x, 0)=u_{0}(x) \in L^{1}\left(\mathbf{R}^{n}\right)$, and the associated nonlinear Fokker-Planck equation (NFPE):

$$
\frac{\partial p}{\partial \tau}=\nabla \cdot\left(\beta x p+D \nabla p^{m}\right), \quad \beta>0
$$

with nonnegative initial data $0 \leq p(x, 0)=p_{0}(x) \in L^{1}\left(\mathbf{R}^{n}\right)$. Here, $D$ is a real symmetric positive definite matrix, which represents the diffusion coefficients. As is widely known [47/48 and shown later, solutions of the both equations are related with a simple transformation.

The PME and NFPE with $m>1$ represent the socalled slow diffusion phenomena, which naturally arises in many physical problems including percolation of a fluid through porous media and so on. See for [32:33|34|35|36 and the references therein. Hence the behaviors of their solutions have been extensively studied in both analytical and thermostatistical aspects in the literature $37 / 38 \mid 39] 40$ just to name a few.

In this section we demonstrate that several geometric concepts derived with generalized entropies in the previous section are useful to investigate a new aspects of the behavior of the above equations. For the proofs of the results see [16].

\subsection{Several geometric properties of the porous medium and the associated Fokker-Planck equation}

Set $\phi(u)=u^{q}, q>0, q \neq 1$, then we have the $q$-logarithmic and exponential functions [51]:

$$
\begin{aligned}
\ln _{\phi} t=\ln _{q} t & :=\left(t^{1-q}-1\right) /(1-q), \\
\exp _{\phi} t=\exp _{q} t & :=[1+(1-q) t]_{+}^{1 /(1-q)} .
\end{aligned}
$$

Consider the $q$-Gaussian density function defined by:

$$
\begin{gathered}
f(x ; \theta, \Theta)=\exp _{q}\left(\theta^{T} x+x^{T} \Theta x-\psi(\theta, \Theta)\right), \\
\theta=\left(\theta^{i}\right) \in \mathbf{R}^{n}, \Theta=\left(\theta^{i j}\right) \in \mathbf{R}^{n \times n}
\end{gathered}
$$

where $\Theta$ is a real symmetric negative definite matrix and $\psi(\theta, \Theta)$ is a normalizing constant. We denote by $\mathcal{M}$ the set of $q$-Gaussian densities, i.e.,

$$
\mathcal{M}:=\left\{f(x ; \theta, \Theta) \mid \theta \in \mathbf{R}^{n}, 0>\Theta=\Theta^{T} \in \mathbf{R}^{n \times n}\right\} .
$$

For this setting, the corresponding generalized entropy and divergence are

$$
\mathcal{I}[p]=\frac{1}{2-q} \int \frac{p(x)^{2-q}-p(x)}{q-1} d x
$$


$\mathcal{D}[p \| r]=\int \frac{r(x)^{2-q}-p(x)^{2-q}}{2-q}-p(x) \frac{r(x)^{1-q}-p(x)^{1-q}}{1-q} d x$

In the sequel we fix the relation between the exponents of the PME and the parameter of $q$-exponential function by $m=2-q$. Hence, we consider the case $1<m<2$, or equivalently, $0<q<1$. Since we fix $\phi(u)=u^{q}$, we omit the subscripts $\phi$ used to denote several quantities. By a suitable linear scaling of $t$ we can consider the problem by fixing $\beta$ to an arbitrary constant. Hence, we fix $\beta$ and introduce another constant $\mu$ for notational simplicity as follows:

$$
\beta:=\frac{1}{n(m-1)+2}, \quad \mu:=n \beta .
$$

For the $q$-Gaussian family $\mathcal{M}$, we can regard $(\theta, \Theta)$ as the canonical coordinates, and the first moment vector and second moment matrix $(\eta, H)$ defined by

$$
\eta:=\int x f(x ; \theta, \Theta) d x, \quad H:=\int x x^{T} f(x ; \theta, \Theta) d x,
$$

as the expectation coordinates, respectively.

We assume the $u(x, 0)$ and $p(x, 0)$ are nonnegative and integrable function with finite second moments. When we consider the set of solutions, we restrict their initial masses to be normalized to one without loss of generalities.

It is proved that there exists a unique nonnegative weak solution if $m>0$ [47, Theorem 5.1], and that the mass $\int u(x, t) d x$ is invariant for all $t>0$ if $m \geq(n-2) / n$ 47.

First of all, we review how the solutions of the PME and NFPE relate in the proposition below. Because of this fact the properties of the solution of the PME (41) are important to investigate those of the NFPE (42) and vise versa.

Proposition 6 Let $u(x, t)$ be a solution of the PME (41) with initial data $u(x, 0)=u_{0}(x) \in L^{1}\left(\mathbf{R}^{n}\right)$. Define

$p(z, \tau):=(t+1)^{\mu} u(x, t), \quad z:=(t+1)^{-\beta} R x, \tau:=\ln (t+1)$, then $p(z, \tau)$ is a solution of (42) with $\nabla=\nabla_{z}, D=R R^{T}$ and initial data $p(z, 0)=u_{0}\left(R^{-1} z\right)$.

Next, we find that the equilibrium density for the NFPE is on the $q$-Gaussian family $\mathcal{M}$ via Lyapunov approach. To analyze the behavior of (42) let us define generalized free energy:

$$
\mathcal{F}[p]:=\int \frac{\beta}{2 m} x^{T} D^{-1} x p(x) d x-\mathcal{I}[p]
$$

This type of functional was first introduced in [39/40. We have

$$
\frac{d \mathcal{F}[p(x, \tau)]}{d \tau}=-\frac{1}{m} \int p\left\|\beta R^{-1} x+m p^{m-2} R \nabla p\right\|^{2} d x \leq 0 .
$$

Thus, the equilibrium density $p_{\infty}(x)$ is determined from (477) as a $q$-Gaussian:

$$
p_{\infty}(x)=f\left(x ; 0, \Theta_{\infty}\right)=\exp _{q}\left(x^{T} \Theta_{\infty} x-\psi\left(0, \Theta_{\infty}\right)\right),
$$

where the canonical parameters are given by

$$
\theta_{\infty}=0, \quad \Theta_{\infty}=-\frac{\beta}{2 m} D^{-1} .
$$

Note that we can express the difference of the free energies of $p(x)$ and the equilibrium $p_{\infty}(x) \in \mathcal{M}$ by the divergence:

$$
\begin{aligned}
\mathcal{D}\left[p \| p_{\infty}\right] & =\Psi\left(0, \Theta_{\infty}\right)-\mathcal{I}[p]-\Theta_{\infty} \cdot \mathbf{E}_{p}\left[x x^{T}\right] \\
& =\mathcal{F}[p]-\mathcal{F}\left[p_{\infty}\right] .
\end{aligned}
$$

Thus, the minimization of $\mathcal{F}[\cdot]$ is equivalent to that of $\mathcal{D}\left[\cdot \| p_{\infty}\right]$.

Finally, we show one of the fundamental properties of the PME and NFPE, which is important to state the sequel results in this paper.

Proposition $\mathbf{7}$ The q-Gaussian family $\mathcal{M}$ is an invariant manifold for both PME and NFPE.

\subsection{Trajectories of $\mathbf{m}$-projections}

Let $\eta^{\mathrm{PM}}=\left(\eta_{i}^{\mathrm{PM}}\right)$ and $H^{\mathrm{PM}}=\left(\eta_{i j}^{\mathrm{PM}}\right)$ be, respectively, the first moment vector and the second moment matrix, i.e.,

$$
\eta_{i}^{\mathrm{PM}}(t):=\mathbf{E}_{u}\left[x_{i}\right]=\int x_{i} u(x, t) d x, \quad \eta_{i j}^{\mathrm{PM}}(t):=\mathbf{E}_{u}\left[x_{i} x_{j}\right] .
$$

Theorem 1 Consider solutions of the PME with the common initial first and second moments. Then their m-projections to $\mathcal{M}$ evolve monotonically along with the common $m$ geodesic curve that starts from the density determined by the initial moments.

Outline of the proof) Differentiating $\eta_{i j}^{\mathrm{PM}}$ by $t$, we see that the second moments evolves as

$$
\begin{aligned}
\eta_{i j}^{\mathrm{PM}}(t) & =\eta_{i j}^{\mathrm{PM}}(0)+\delta_{i j} \sigma_{u}^{\mathrm{PM}}(t), \\
\sigma_{u}^{\mathrm{PM}}(t) & :=2 \int_{0}^{t} d t^{\prime} \int u\left(x, t^{\prime}\right)^{m} d x .
\end{aligned}
$$

Note that $\sigma_{u}^{\mathrm{PM}}(t)$ is positive and monotone increasing on $t>0$. By similar argument we see that $\dot{\eta}_{i}^{\mathrm{PM}}=0$, i.e., the first moment vector is invariant. From Proposition 4 and i) of Proposition 5, the statement follows.

Remark 2 i) From the argument for the NFPE, we will see that $\sigma_{u}^{\mathrm{PM}}(t)=O\left(t^{2 \beta}\right)$ as $t \rightarrow \infty$.

ii) The theorem implies that the trajectories of m-projections on $\mathcal{M}$ for all the PME solutions $u(x, t)$ are parallelized in the expectation coordinates, i.e.,

$$
\begin{aligned}
\eta^{\mathrm{PM}}(t) & =\eta^{\mathrm{PM}}(0), \\
H^{\mathrm{PM}}(t) & =H^{\mathrm{PM}}(0)+\sigma_{u}^{\mathrm{PM}}(t) I,
\end{aligned}
$$

where $I$ denotes the identity matrix. See [16] for the argument on the constants of motion. 


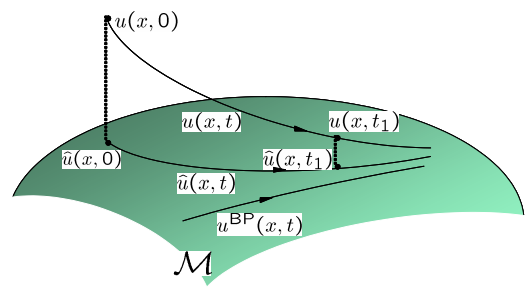

Fig. 2. A solution $u(x, t)$ of the PME, its m-projection $\hat{u}(x, t)$ and the Barenblatt-Pattle solution $u^{\mathrm{BP}}(x, t)$ on $\mathcal{M}$.

Let $\hat{f}_{0}(x) \in \mathcal{M}$ be the m-projection of the density $f_{0}(x)$. Consider two solutions $u_{1}(x, t)$ and $u_{2}(x, t)$ of the PME satisfying $u_{1}\left(x, t_{0}\right)=f_{0}(x)$ and $u_{2}\left(x, t_{0}\right)=\hat{f}_{0}(x)$ for a certain $t_{0}$. From the moment conservation property of the m-projection stated in Proposition 5, the second moment matrices $H_{i}^{\mathrm{PM}}(t)$ of $u_{i}(x, t)$ for $i=1,2$ satisfy $H_{1}^{\mathrm{PM}}\left(t_{0}\right)=H_{2}^{\mathrm{PM}}\left(t_{0}\right)$. However, their velocities at $t_{0}$ have the relation:

$$
\begin{aligned}
\dot{H}_{1}^{\mathrm{PM}}\left(t_{0}\right)-\dot{H}_{2}^{\mathrm{PM}}\left(t_{0}\right) & =2 \int f_{0}^{m}(x)-\hat{f}_{0}^{m}(x) d x I \\
& =2 m(m-1)\left(\mathcal{I}\left[\hat{f}_{0}\right]-\mathcal{I}\left[f_{0}\right]\right) I
\end{aligned}
$$

from (50) and the expression of the generalized entropy (45). Using the relation in Remark 1, we have the following:

Corollary 1 Let $\hat{f}_{0}(x) \in \mathcal{M}$ be the m-projection of a density $f_{0}(x)$ and assume that two solutions $u_{1}(x, t)$ and $u_{2}(x, t)$ of the PME satisfy the conditions $u_{1}\left(x, t_{0}\right)=f_{0}(x)$ and $u_{2}\left(x, t_{0}\right)=\hat{f}_{0}(x)$ at $t=t_{0}$. Then velocities of their respective second moment matrices at $t_{0}$ are related by

$$
\dot{H}_{1}^{\mathrm{PM}}\left(t_{0}\right)-\dot{H}_{2}^{\mathrm{PM}}\left(t_{0}\right)=2 m(m-1) \mathcal{D}\left[f_{0} \| \hat{f}_{0}\right] I .
$$

Thus, the m-projection $\hat{u}_{1}(x, t)$ of $u_{1}(x, t) \notin \mathcal{M}$, which has the common second moment matrix $H_{1}^{\mathrm{PM}}(t)$ for all $t$, evolves faster than $u_{2}(x, t) \in \mathcal{M}$, while $\hat{u}_{1}(x, t)$ and $u_{2}(x, t)$ have the common trajectory on $\mathcal{M}$ by Theorem 1 (See Figure 3). The corollary suggests that by measuring the diagonal elements of $H_{1}^{\mathrm{PM}}(t)$ we can estimate how far $u_{1}(x, t)$ is from $\mathcal{M}$ in terms of the divergence. Note that the difference of velocities vanishes when $m \rightarrow 1$. Hence, this is the specific property of the slow diffusions governed by the PME.

Let $\eta^{\mathrm{FP}}(\tau)$ and $H^{\mathrm{FP}}(\tau)$ be, respectively, the first and the second moments of $p(x, \tau)$, i.e.,

$$
\eta^{\mathrm{FP}}=\mathbf{E}_{p}[x], \quad H^{\mathrm{FP}}=\mathbf{E}_{p}\left[x x^{T}\right] .
$$

From the behavior of the moments of the PME and the above relations of moments, we have

$$
\begin{aligned}
\eta^{\mathrm{FP}}(\tau) & =e^{-\beta \tau} \eta^{\mathrm{FP}}(0), \\
H^{\mathrm{FP}}(\tau) & =e^{-2 \beta \tau} H^{\mathrm{FP}}(0)+e^{-2 \beta \tau} \sigma_{p}^{\mathrm{FP}}\left(e^{\tau}-1\right) D,
\end{aligned}
$$
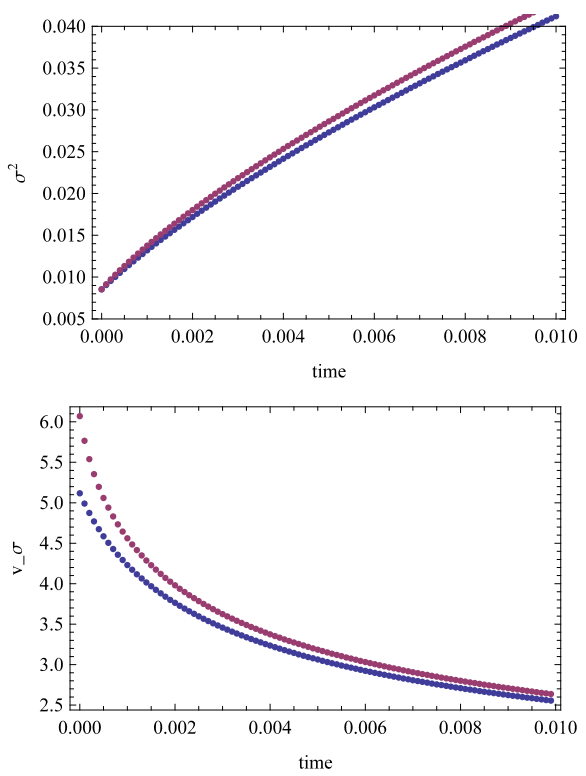

Fig. 3. Evolutions of the second moments of $u_{1}(x, t) \notin \mathcal{M}$ and $u_{2}(x, t) \in \mathcal{M}$ with the same initial moments for the onedimensional PME $(m=1.9)$ (Above) and the corresponding velocities (Below).

where the scaling $\tau=\ln (t+1)$ is assumed and $\sigma_{p}^{\mathrm{FP}}(t)$ is defined by

$$
\begin{aligned}
\sigma_{p}^{\mathrm{FP}}(t) & :=2 \int_{0}^{\ln (1+t)} d \tau^{\prime} e^{\tau^{\prime}+\mu(1-m) \tau^{\prime}} \int p\left(x, \tau^{\prime}\right)^{m} d x \\
& =\operatorname{det}(R) \sigma_{u}^{\mathrm{PM}}(t) .
\end{aligned}
$$

for a solution $u$ of the PME and the corresponding solution $p$ of the NFPE. Note that differentiating the above by $t$, we have the relation:

$$
(1+t)^{\mu(1-m)} \int p(z, \tau)^{m} d z=\operatorname{det}(R) \int u(x, t)^{m} d x .
$$

For the limiting case $m \rightarrow 1$ (and accordingly $\beta \rightarrow$ $1 / 2$ ), we see that the above expressions recover the wellknown linear Fokker-Plank case with a drift vector $x / 2$ :

$$
\begin{aligned}
\eta^{\mathrm{FP}}(\tau) & =e^{-\tau / 2} \eta^{\mathrm{FP}}(0), \\
H^{\mathrm{FP}}(\tau) & =e^{-\tau} H^{\mathrm{FP}}(0)+2\left(1-e^{-\tau}\right) D .
\end{aligned}
$$

Since we know that $p(x, \tau)$ converges to $p_{\infty}(x) \in \mathcal{M}$ in (48) and it holds that

$$
\lim _{\tau \rightarrow \infty} H^{\mathrm{FP}}(\tau)=\sqrt{\operatorname{det} D}\left(\lim _{t \rightarrow \infty}(t+1)^{-2 \beta} \sigma_{u}^{\mathrm{PM}}(t)\right) D
$$

because $\operatorname{det} R=\sqrt{\operatorname{det} D}$, we conclude that the left-hand side of (52) exists and $\sigma_{u}^{\mathrm{PM}}(t)=O\left(t^{2 \beta}\right)$ as $t \rightarrow \infty$ (Cf. Remark 2). Summing up the above with Proposition 4 , we obtain the following geometric property of the NFPE:

Corollary 2 Consider solutions of the NFPE with the common initial first and second moments. Then their mprojections to $\mathcal{M}$ evolve along with the common m-geodesic 
curve approaching from the density determined by the initial moments to the equilibrium $p_{\infty}(x)$.

\subsection{Convergence rate of the solution of the PME to $\mathcal{M}$}

The following proposition is obtained from ii) of Proposition 5 and a result 4548 claiming that a solution of the NFPE decays exponentially with respect to the divergence, i.e.,

$$
\mathcal{D}\left[p(x, \tau) \| p_{\infty}(x)\right] \leq \mathcal{D}\left[p(x, 0) \| p_{\infty}(x)\right] e^{-2 \beta \tau} .
$$

Proposition 8 Let $u(x, t)$ be a solution of the PME and $\hat{u}(x, t)$ be the m-projection of $u(x, t)$ to the q-Gaussian family $\mathcal{M}$ at each $t$. Then $u(x, t)$ asymptotically approaches to $\mathcal{M}$ with

$$
\mathcal{D}[u(x, t) \| \hat{u}(x, t)] \leq \frac{C_{0}}{1+t},
$$

where $C_{0}$ is a constant depending on the initial function $u(x, 0)$.

Remark 3 Combining this result and the Csiszar-Kullback inequality 45, we can also conclude the $L^{1}$ convergence of $u(x, t)$ to $\mathcal{M}$ with the rate $1 / \sqrt{1+t}$. This implies that the convergence to $\mathcal{M}$ is faster than $1 / t^{\beta}$, which is the convergence rate to the self-similar solution of the PME in the case $1<m \leq 2$ 47/48.

\section{Conclusions}

We have discussed the Legendre duality of generalized entropies and its applications focusing on the duality relation (1) of the statistical manifold. Within this framework, we classified extensions into two major directions using the representation functions. In terms of corresponding geometric structure, we can say that the one is characterized by nonflatness with constant curvature, while the other is by dual flatness.

The important point would be how such a geometric setup is useful for our understandings of various physical phenomena not obeying the standard statistical theory. For this purpose we have partially presented the recent results on the solutions of the PME and the NFPE. However, the whole picture of the proposed generalization in Section 5 is still largely formal and it needs more developments on the basis of physical background.

\section{References}

1. C. Beck and F. Schlögl, Thermodynamics of Chaotic Systems, (Cambridge University Press, Cambridge, 1993).

2. C. Tsallis, Physica D, 193, 3 (2004).

3. T. D. Frank, Nonlinear Fokker-Planck Equations: Fundamentals and Applications, (Springer-Verlag, 2005).

4. P. H. Chavanis, Eur. Phys. J. B, 62, 179 (2008).

5. S-I. Amari, Differential-Geometrical Methods in Statistics, (Springer-Verlag, Berlin 1985).
6. S-I. Amari and H. Nagaoka, Methods of Information Geometry, (AMS \& Oxford, Rhode Island 2000).

7. T. Kurose, Math. Z., 203, 115 (1990).

8. T. Kurose, Tôhoku Math. J., 46, 427 (1994).

9. K. Nomizu and T. Sasaki, Affine Differential Geometry, (Cambridge University Press, Cambridge 1994).

10. L. M. Bregman, USSR comp. math. math. phys., 7, 200 (1967).

11. S. Eguchi, Sugaku Expositions, 19, 197 (2006), (originally Sūgaku, 56, 380 (2004) in Japanese.)

12. N. Murata, T. Takenouchi, T. Kanamori and S. Eguchi, Neural Computation, 16, 1437 (2004).

13. J. Naudts, Physica A, 316, 323 (2002).

14. J. Naudts, Physica A, 340, 32 (2004).

15. J. Naudts, J. Ineq. Pure Appl. Math, 5, 4, 102 (2004).

16. A. Ohara and T. Wada, [arXiv: 0810.0624v1].

17. J. H. Havrda and F. Charvat, Kybernetika, 3, 30 (1967).

18. C. Tsallis, J. Stat. Phys., 52, 479 (1988).

19. P. N. Rathie and Pl. Kannappan, Information and Control, 20, 38 (1972).

20. J. Aczél and Z. Daróczy, On measures of information and their characterizations, (Academic Press, New York 1975).

21. L. Borland, A. R. Plastino and C. Tsallis, J. Math. Phys., 39, 6490 (1998).

22. M. Shiino, J. Phys. Soc. Jpn., 67, 3658 (1998).

23. A. Ohara, Phys. Lett. A, 370, 184 (2007).

24. J. H. Hao and H. Shima, Geom. Dedicata, 50, 193 (1994).

25. H. Shima, Geom. Dedicata, 56, 177 (1995).

26. K. Uohashi, A. Ohara and T. Fujii, Osaka J. Math., 37, 501 (2000).

27. C. Tsallis, R. S. Mendes and A. R. Plastino, Physica A, 261, 534 (1998).

28. A. Fujiwara and S-I. Amari, Physica D, 80, 317 (1995).

29. P. D. Grunwald and A. P. David, Annals of Statistics 32, 1367-1433 (2004).

30. H. B. Callen, Thermodynamics and an Introduction to Thermostatistics, second ed., (Wiley, New York 1985).

31. T. Wada and A. M. Scarfone, Phys. Lett. A, 335, 351 (2005).

32. M. Muskat, The Flow of Homogeneous Fluids Through Porous Media, (McGraw-Hill, New York 1937).

33. J. Buckmaster, J. Fluid Mech., 81, 735 (1977).

34. E. W. Larsen and G. C. Pomraning, SIAM J. Appl. Math., 39, 201 (1980).

35. W. L. Kath, Physica D, 12, 375 (1985).

36. G. I. Barenblatt, Scaling, self-similarity and intermediate asymptotics, (Cambridge Univ. Press, Cambridge, 1996).

37. A. Friedman and S. Kamin, Trans. Amer. Math. Soc., 262, 551 (1980).

38. D. G. Aronson, The Porous Medium Equation, (SpringerVerlag, Berlin/New York 1985).

39. W. Newman, J. of Math. Phys., 25, 3124 (1984).

40. J. Ralston, J. of Math. Phys., 25, 3120 (1984).

41. A. R. Plastino and A. Plastino, Physica A, 222, 347 (1995).

42. M. Shiino, J. Math. Phys., 42, 2540 (2001).

43. T. D. Frank, Physics Letters A, 290, 93 (2001).

44. T. D. Frank, Physica A, 310, 397 (2002).

45. J. A. Carrillo and G. Toscani, Indiana Univ. Math. J., 49, $113(2000)$.

46. F. Otto, Comm. Partial Differential Equations, 26, 101 (2001).

47. J. L. Vázquez, J. Evol. Equ., 3, 67 (2003). 
48. G. Toscani, J. Evol. Equ., 5, 185 (2005).

49. G. I. Barenblatt, Prikl. Mat. Mekh., 16, 67 (1952) (in Russian).

50. R. E. Pattle, Quart. Jour. Mech. Appl. Math., 12, 407 (1959).

51. C. Tsallis, S. V. F. Levy, A. M. C. Souza and R. Maynard, Phys. Rev. Lett., 75, 3589 (1995), Errata, 77, 5442 (1996).

\section{Appendix}

\section{A Statistical manifolds and affine differential geometry}

In this appendix we briefly summarize several concepts and properties of statistical manifolds and affine hypersurfaces, which are necessary in this paper. See for details 789, respectively.

\section{A.1 Statistical manifold}

For a torsion-free affine connection $\nabla$ and a pseudo Riemannian metric $g$ on a manifold $\mathcal{M}$, the triple $(\mathcal{M}, g, \nabla)$ is called a statistical manifold if it admits another torsionfree connection $\nabla^{*}$ satisfying

$$
X g(Y, Z)=g\left(\nabla_{X} Y, Z\right)+g\left(Y, \nabla_{X}^{*} Z\right)
$$

for arbitrary $X, Y$ and $Z$ in $\mathcal{X}(\mathcal{M})$, where $\mathcal{X}(\mathcal{M})$ is the set of all tangent vector fields on $\mathcal{M}$. We call $\nabla$ and $\nabla^{*}$ duals of each other with respect to $g$, and $\left(\mathcal{M}, g, \nabla^{*}\right)$ is said dual statistical manifold of $(\mathcal{M}, g, \nabla)$. The triple of a Riemannian metric and a pair of dual connections $\left(g, \nabla, \nabla^{*}\right)$ satisfying (54) is called dualistic structure on $\mathcal{M}$, which plays a fundamental role in the study of manifolds of probability distributions.

A statistical manifold $(\mathcal{M}, g, \nabla)$ is said to have constant curvature $\kappa$ if the curvature tensor $R$ of $\nabla$ satisfies

$$
R(X, Y) Z=\kappa\{g(Y, Z) X-g(X, Z) Y\} .
$$

When the constant $\kappa$ is zero, the statistical manifold is said to be flat, or dually flat, because the curvature tensor $R^{*}$ of $\nabla^{*}$ is known to vanish automatically.

\section{A.2 Affine hypersurface theory}

Let $\mathcal{M}$ be an $n$-dimensional manifold and consider an affine immersion $(f, \xi)$, which is the pair of an immersion $f$ of $\mathcal{M}$ into $\mathbf{R}^{n+1}$ and a transversal vector field $\xi$ to $f(\mathcal{M})$. We denote by $D_{X} f_{*}(Y)$ the covariant derivative along $f$ induced by the standard flat connection $D$ of $\mathbf{R}^{n+1}$. By a given affine immersion $(f, \xi)$ of $\mathcal{M}$, the Gauss and Weingarten formulas are respectively obtained as follows:

$$
\begin{aligned}
D_{X} f_{*}(Y) & =f_{*}\left(\nabla_{X} Y\right)+h(X, Y) \xi \\
D_{X} \xi & =-f_{*}(S X)+\tau(X) \xi
\end{aligned}
$$

Here, $\nabla, h, S$ and $\tau$ determined from the above formulas are called, respectively, the induced connection, affine fundamental form, affine shape operator and transversal connection form 9. By regarding $h$ as a (pseudo-) Riemannian metric of $\mathcal{M}$, we say that the affine immersion realizes $(\mathcal{M}, h, \nabla)$ in $\mathbf{R}^{n+1}$.

An affine immersion is said nondegenerate and equiaffine if $h$ is nondegenerate and $\tau=0$, respectively. Further, let $o$ be the origin of $\mathbf{R}^{n+1}$. Then we say that the affine immersion is centro-affine with a scaling constant $\rho$ if $\xi$ at $f(x)$ is equal to $-\rho$ times of the vector $\overrightarrow{o f(x)}$ for a constant $\rho$ and $x \in \mathcal{M}$.

The following facts hold [9], which are convenient to know the property of the realized manifold by an affine immersion:

F1) An equiaffine and nondegenerate affine immersion realizes a statistical manifold,

F2) A centro-affine immersion with a scaling constant $\rho$ is equiaffine with $S=\rho I$. The realized manifold is projectively flat,

F3) An equiaffine and nondegenerate affine immersion with $S=\rho I$ realizes a statistical manifold with constant curvature $\rho$.

Let $(\mathcal{M}, g, \nabla)$ be a statistical manifold with constant curvature, which is realized by a centro-affine immersion with constant scaling. It is known that $(\mathcal{M}, g, \nabla)$ has the following properties [89]:

P1) Modified Pythagorean relation for the geometric divergence (contrast function) holds on $\mathcal{M}$,

P2) Let $\gamma$ be a $\nabla$-geodesic curve joining two points $x$ and $y$ on $\mathcal{M}$. Then, $f(\gamma)$ is the intersection of $f(\mathcal{M})$ and the two-dimensional plane containing $\overrightarrow{o f(x)}$ and $\overrightarrow{o f(y)}$ in $\mathbf{R}^{n+1}$.

The corresponding results also hold for the dual statistical manifold $\left(\mathcal{M}, g, \nabla^{*}\right)$ with constant curvature.

\section{B Proof of Proposition 3}

First, recall that $D^{(\alpha)}$ is the restriction of $\mathcal{D}$ in (22) defined on $\mathbf{R}_{+}^{n+1} \times \mathbf{R}_{+}^{n+1}$. Hence, in the ambient space $\left(\mathbf{R}_{+}^{n+1}, \tilde{g}, D\right)$, the gradient vector $\tilde{X}$ of $\mathcal{D}(\boldsymbol{\theta}, \boldsymbol{\theta}(\boldsymbol{r}))$ at $\boldsymbol{\theta} \in \mathbf{R}_{+}^{n+1}$ is represented by

$$
\tilde{X}=\sum_{i=1}^{n+1} \tilde{X}^{i} \frac{\partial}{\partial \theta^{i}}
$$

where

$$
\begin{aligned}
\tilde{X}^{i} & =-\sum_{j=1}^{n+1} \tilde{g}^{i j} \frac{\partial}{\partial \theta^{j}} \mathcal{D}(\boldsymbol{\theta}, \boldsymbol{\theta}(\boldsymbol{r})) \\
& =\sum_{j=1}^{n+1} \tilde{g}^{i j}\left(\eta_{j}(\boldsymbol{r})-\eta_{j}\right), i=1, \cdots, n+1,
\end{aligned}
$$

and $\tilde{g}^{i j}$ is the component of the inverse of the Riemannian metric $\tilde{g}=\left(\partial^{2} \psi / \partial \theta^{i} \partial \theta^{j}\right)$ given in (25). Hence, the 
gradient vector $\tilde{X}$ is represented in the $\eta$-coordinates by

$$
\tilde{X}=\sum_{i=1}^{n+1} \tilde{X}_{i} \frac{\partial}{\partial \eta_{i}}, \quad \tilde{X}_{i}=\eta_{i}(\boldsymbol{r})-\eta_{i}
$$

and its integral curve is

$$
\eta_{i}(\boldsymbol{p}(t))=e^{-t}\left(\eta_{i}\left(\boldsymbol{p}_{0}\right)-\eta_{i}(\boldsymbol{r})\right)+\eta_{i}(\boldsymbol{r}) .
$$

Next, consider the vector field defined by

$$
N=\sum_{i=1}^{n+1} p_{i} \frac{\partial}{\partial p_{i}}=\sum_{i=1}^{n+1} \frac{1-\alpha}{2} \theta^{i} \frac{\partial}{\partial \theta^{i}}=\sum_{i=1}^{n+1} \frac{1+\alpha}{2} \eta_{i} \frac{\partial}{\partial \eta_{i}}
$$

then $N$ is orthogonal to $T_{p} \mathcal{S}^{n}$ at each $\boldsymbol{p} \in \mathcal{S}^{n}$ with respect to $\tilde{g}$. It is verified that the gradient vector $X$ is represented as the orthogonal projection of $\tilde{X}$ onto $T_{p} \mathcal{S}^{n}$ along $N$.

Combining the above two facts, we see that the integral curve (gradient flow) of $X$ is restricted to the twodimensional plane $\mathcal{P}^{*}$ containing the three points $\boldsymbol{\eta}\left(\boldsymbol{p}_{0}\right)$, $\boldsymbol{\eta}(\boldsymbol{r})$ and the origin $o$ in $\left(\mathbf{R}^{n+1}\right)^{*}$ with the $\eta$-coordinate system. Thus, the second statement follows. Note that $\mathcal{S}^{n}$ is represented as the level surface $\varphi(\boldsymbol{\eta})=2 /(1-\alpha)$ in $\left(\mathbf{R}^{n+1}\right)^{*}$, then we conclude that the gradient flow is actually represented by the intersection of the level surface and $\mathcal{P}^{*}$. This proves the first statement owing to the property (P2) in Appendix A for the dual statistical manifold $\left(\mathcal{S}^{n}, g, \nabla^{(-\alpha)}\right)$. 